\title{
Ionisation und Dissoziation durch Elektronenstoß bei Methan, Methylalkohol und Methylal
}

\author{
Von J. Geerk und H. Neuert * \\ (Z. Naturforschg. 5 a, 502-504 [1950]; eingegangen am 13. September 1950)
}

\begin{abstract}
Zur genaueren Kenntnis der Ionenarten und -häufigkeiten, die z. B. bei den Entladungen in Zählrohren mit Dampffüllung (Alkoholdampf und ähnliche Kohlenwasserstoffe) auftreten können, wurden Methan, Methylalkohol und Methylal im Massenspektrometer untersucht. Dabei wurden die relativen Häufigkeiten der wichtigsten Ionensorten in ihrer Abhängigkeit von der Elektronenenergie im Bereich bis zu $100 \mathrm{eV}$ gemessen und die kritischen Potentiale für Dissoziation, soweit noch nicht bekannt, bestimmt. Es zeigte sich, daß in den Entladungen auch bei den schwereren Dämpfen praktisch nur die aus Dissoziationsprozessen stammenden Icnen mittlerer oder geringer Massenzahl eine Rolle spielen.
\end{abstract}

$\mathrm{F}$ ür das Studium von Entladungsvorgängen in Zählrohren mit reiner Dampffüllung oder mit Gasfüllung mit Dampfzusatz ist es wichtig zu wissen, welche Ionenarten in den Entladungslawinen durch Elektronenstoß oder durch Photoionisation entstehen. Die Ionisations- und Dissoziationsprozesse durch sorte gerade zum ersten Male auftritt (Ionisationspotential IP oder kritisches Potential der Dissoziation durch Elektronenstoß = appearance potential $A P$ ). Messungen der relativen Häufigkeiten der verschiedenen Ionensorten sind bei einigen der für das Zählrohr interessierenden Substanzen schon früher ge-
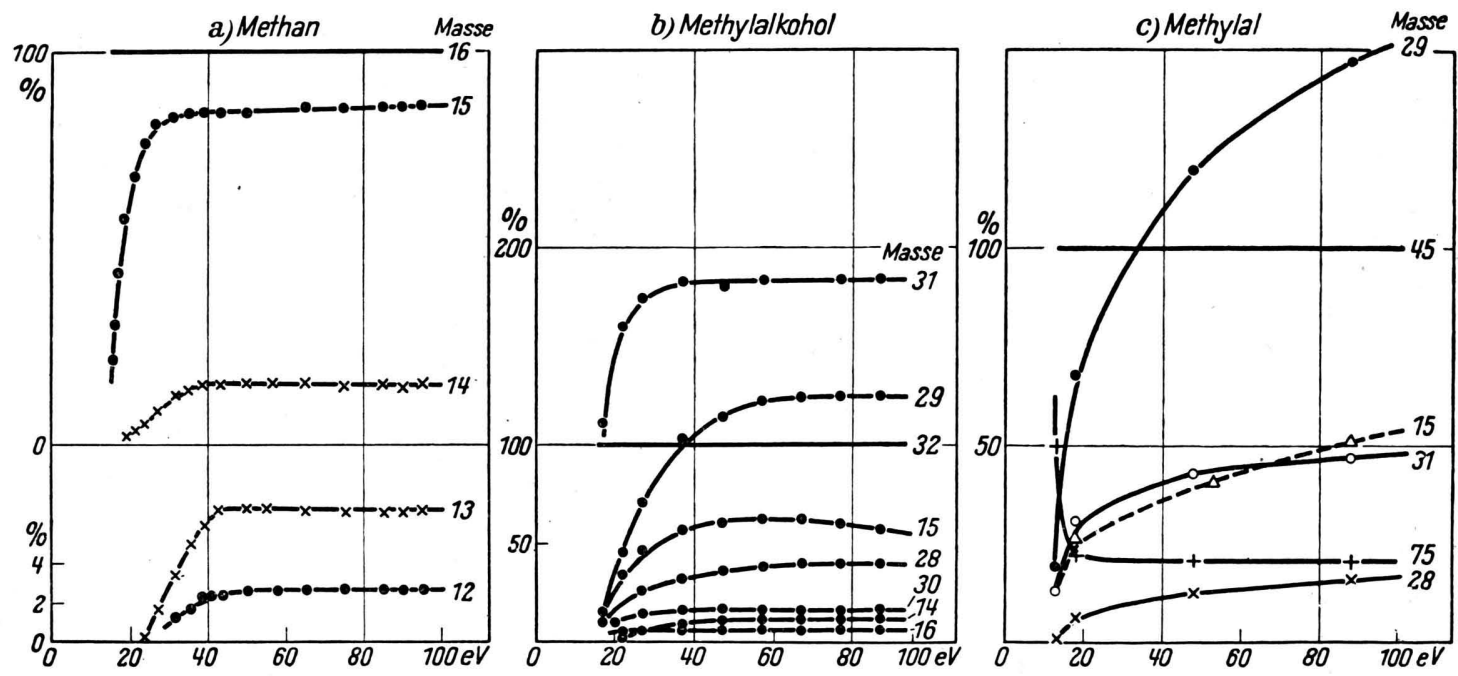

Abb. 1. Relative Häufigkeiten der wichtigsten Dissoziationsprodukte als Funktion der Elektronenenergie bei: a) Methan, b) Methylalkohol, c) Methylal. Ordinate: Ionenhäufigkeit a) relativ zur Masse 16, b) relativ zur Masse 32, c) relativ zur Masse 45. Abszisse: Elektronerenergie.

Elektronenstoß lassen sich, gerade in dem bei Zählrohren interessierenden Bereich der Elektronenenergien bis etwa $40 \mathrm{eV}$ verhältnismäßig einfach mit dem Massenspektrometer untersuchen. Aus derartigen Versuchen erhält man ferner Auskunft, bei welchen Mindestenergien der Elektronen eine betrachtete Ionen-

* (17 b) Weil a. Rh., Pfädlistr. 12, Marktplatz 5.

1 L. G. S m it h, Physic. Rev. 51, 263 [1937]. macht worden, allerdings meist nur für eine feste Elektronenenergie, z. B. für 70 oder $100 \mathrm{eV}$. Die schon bekannten Resultate an Methan $^{1}$ und an Methylalkohol ${ }^{2}$ wurden überprüft und die Messungen der relativen Häufigkeiten der Ionen auf den gesamten Energiebereich der Elektronen bis $100 \mathrm{eV}$

2 C. S. Cummings u. W. B leakney, Physic. Rev. 58, 787 [1940]. 


\begin{tabular}{|l|c|c|c|c|c|c|}
\hline & & & & & & \\
Masse . . . . . & 16 & 15 & 14 & 13 & 12 & 1 \\
Ion. . . . . . & $\mathrm{CH}_{4}^{+}$ & $\mathrm{CH}_{3}^{+}$ & $\mathrm{CH}_{2}^{+}$ & $\mathrm{CH}^{+}$ & $\mathrm{C}^{+}$ & $\mathrm{H}^{+}$ \\
Nach Smith . . & $13,1 \pm 0,4$ & $14,4 \pm 0,4$ & $15,7 \pm 0,5$ & $23,3 \pm 0,6$ & $26,7 \pm 0,7$ & $22,7 \pm 0,5$ \\
Hier gefundene & & & $22,1 \pm 0,8$ & & & $29,4 \pm 0,6$ \\
Werte. . . . & $13,3 \pm 0,3$ & $14,5 \pm 0,3$ & $16,5 \pm 0,5$ & $22,5 \pm 0,5$ & $21,5 \pm 1$ & - \\
& & & $21,5 \pm 1,0$ & $27,0 \pm 1,0$ & $29,5 \pm 1$ & - \\
\hline
\end{tabular}

Tab. 1. IP- und AP-Werte bei Methan in eV.

\begin{tabular}{|lll|c|c|c|c|c|c|}
\hline Masse . . . . &. & 75 & 45 & 31 & 29 & 28 & 15 \\
Ion . . . . . &. & $\mathrm{C}_{3} \mathrm{H}_{7} \mathrm{O}_{2}^{+}$ & $\mathrm{C}_{2} \mathrm{H}_{5} \mathrm{O}^{+}$ & $\mathrm{CH}_{3} \mathrm{O}^{+}$ & $\mathrm{CHO}^{+}$ & $\mathrm{CO}^{+}$ & $\mathrm{CH}_{3}^{+}$ \\
AP-Wert . . . &. & $11,0 \pm 0,3$ & $11,5 \pm 0,3$ & $13,3 \pm 0,3$ & $13,9 \pm 0,3$ & $13,3 \pm 0,3$ & $13,7 \pm 0,3$ \\
\hline
\end{tabular}

Tab. 2. AP-Werte bei $\mathrm{CH}_{2}\left(\mathrm{OCH}_{3}\right)_{2}$ in $\mathrm{eV}$.

ausgedehnt. Insbesondere wurde die Dissoziation von Methylal studiert, das z. B. nach Trost ${ }^{3}$ für Zählrohrfüllungen besonders geeignet zu sein scheint.

\section{Resultate der Messungen}

Die Untersuchungen wurden mit einem $60^{\circ}$-Massenspektrometer durchgeführt. Die $I P$ - und $A P$-Werte wurden durch Vergleichsmessungen mit Argon gewonnen.

a) $\mathrm{CH}_{4}$

Die relativen Häufigkeiten der wichtigsten Ionensorten bei Methan sind in ihrer Abhängigkeit von der Elektronenenergie in Abb. 1 a aufgetragen. Die von Smith allerdings nur bei einer Elektronenenergie von $50 \mathrm{eV}$ gemessenen Werte stimmen für das Häufigkeitsverhältnis der Massen 15 und 16 innerhalb der Meßgenauigkeit mit den hier gefundenen überein, während für die Massen 12, 13 und 14 dort nur die etwa halben Werte für die Häufigkeiten gemessen worden sind. Die hier gefundenen Werte für das Ionisationspotential und die kritischen Potentiale der Dissoziation sind im Vergleich mit den Werten von Smith in Tab. 1 zusammengestellt.

Die Werte für die Massen 16, 15 und 14 stimmen gut miteinander überein, der Wert für Masse 14 weicht allerdings etwas von dem von $\mathrm{K}$ of $\mathrm{fel}$ und L a d ${ }^{4}$ gemessenen Werte $(20,0 \pm 0,3 \mathrm{eV})$ ab. Bei den Massen 12, 13 und 14 scheinen nach den hier vorliegenden Messungen jeweils zwei verschiedene kritische Potentiale aufzutreten. Mit Sicherheit liegen

3 A. Trost, Z. Physik 105, 399 [1937]; Z. angew. Physik 2, 286 [1950]. die beiden kleineren Werte der Massen 12 und 13 bei geringeren Energien als bei Smith. Die beiden höheren Werte kann man aus den Meßkurven nur unsicher entnehmen. Das Auftreten zweier getrennter AP-Werte bei den Massen 12 und 13 ist, ebenso wie bei der Masse 14, ohne weiteres so zu klären, daß diese Massen bei verschiedenen Dissoziationsprozessen entstehen können. So kann z. B. die Masse 13 beim Prozeß

$$
\mathrm{CH}_{4}+\mathrm{e}^{-} \rightarrow \mathrm{CH}^{+}+3 \mathrm{H}+2 \mathrm{e}^{-}
$$

oder durch

$$
\mathrm{CH}^{+}+\mathrm{H}_{2}+\mathrm{H}+2 \mathrm{e}^{-}
$$

entstehen.

\section{b) $\mathrm{CH}_{3} \mathrm{OH}$}

Die beim Methylalkohol gefundenen Häufigkeiten der wichtigsten Ionensorten sind aus Abb. $1 \mathrm{~b}$ ersichtlich. Die von Cummings und Bleakney bei $70 \mathrm{eV}$ gemessenen Werte lassen sich gut in diese Kurven einfügen, mit Ausnahme des Wertes für die Masse 28 $\left(\mathrm{CO}^{+}\right)$, für welche hier nur eine etwa halb so große Häufigkeit gefunden wurde.

\section{c) $\mathrm{CH}_{2}\left(\mathrm{OCH}_{3}\right)_{2}$}

Methylal ist bisher noch nicht im Massenspektrometer untersucht worden. Die relativen Häufigkeiten der mit größerer Intensität auftretenden Ionensorten als Funktion der Elektronenenergie zeigt Abb. $1 \mathrm{c}$. Außer den dort aufgeführten Massen wurden noch bei den Massenzahlen 47, 46, 44, 32, 27 und 16 nen-

${ }_{4}$ M. B. K of f e l u. R. A. L a d, J. chem. Physics 16, 420 [1948]. 
nenswerte Ionenintensitäten festgestellt. Die Masse 76 des ionisierten Methylalmoleküls ist nicht mit mehr als $1 \%$ der Intensität der Masse 75 beobachtet worden. Die AP-Werte der häufigsten Ionensorten finden sich in Tab. 2.

\section{S chlußbemerkung}

Aus den Messungen geht im Hinblick auf die Häufigkeit der in Gasentladungen durch Elektronenstoß entstehenden Ionen hervor, daß bei zahlreichen Kohlenwasserstoffen mit geringerem Molekulargewicht die Ionen des Ausgangsmoleküls sowie des um ein H-Atom kleineren Moleküls überwiegen. Bei schwereren Molekülen tritt das Ion des Ausgangsmoleküls in vielen Fällen gar nicht mehr, das um die Masse 1 kleinere Ion nur noch mit geringer Intensität in Erscheinung. So hat man es beim Methylal im Zählrohr (bei Elektronenenergien bis etwa $40 \mathrm{eV}$ ) offensichtlich hauptsächlich mit Ionen der Massen 45 und 29 zu tun.

Dies wurde durch Messungen an Äthylacetat bestätigt, bei denen die Masse 88 gar nicht, die Masse 87 nur mit etwa 2\%, dagegen die Massen 43, 28 und 29 mit etwa 31 bzw. 13 bzw. 8,5\% der gesamten Ionenmenge auftraten.

Die hier durchgeführten Messungen können gleichzeitig dazu dienen, den Vorrat an Meßresultaten, die zur Durchführung von Gasanalysen mit dem Massenspektrometer benötigt werden, zu ergänzen.

\title{
Zur Bestimmung der Verschiebungsgeschwindigkeiten von Flächen optisch anisotroper Kristalle
}

\author{
Von Rudolf Mosebach \\ Aus dem Mineralogisch-Petrographischen Institut der Universität Tübingen \\ (Z. Naturforschg. 5 a, 504-507 [1950]; eingegangen am 22. August 1950)
}

\begin{abstract}
Eine mikroskopisch-optische Methode zur Bestimmung der Verschiebungsgeschwindigkeiten $v_{(h k l)}$ von Flächen wachsender, nicht regulärer Kristalle in übersättigten Lösungen wird beschrieb€n. Kristallplättchen, die durch natürliche Flächen oder Spaltflächen planparallel begrenzt sind, zeigen beim Wachstum in übersättigter Lösung häufig planparalleles Fortwachsen durch fiächenmäßige Substanzanlagerung. In diesen Fällen verursacht der Schichtdicken-Zuwachs eine Steigerung des optischen Gangunterschiedes $T$. Dieser Zuwachs läßt sich bei bekannten kristallographischen und optischen Konstanten kompensatorisch quantitativ bestimmen. Versuche an 2 triklin kristallisierenden Substanzen, Kupfervitriol und Kaliumbichromat, zeigen die Anwendbarkeit der Methode. Das stetige Wandern des Kompensationsstreifens erlaubt die zeitliche Verfolgung des Wachstums. Eine Entfernung des wachsenden Kristalls aus seiner Mutterlauge zur Bestimmung seines Dickenzuwachses ist nicht erforderlich, was die Anwendung dieser Methode für viele Zwecke empfiehlt.
\end{abstract}

$\mathrm{U}$ nter den Substanzen, an denen die Erscheinungen des Wachstums und der Auflösung von Kristallen in überbzw. untersättigter Lösung studiert worden sind, finden wir hauptsächlich regulär kristallisierende. Eine Übersicht über die bisher vorliegenden Ergebnisse finden wir bei $\mathrm{S}$ p a n g e $\mathrm{n}$ b e r g 1,2 und $\mathrm{N}$ i g g li ${ }^{3}$. Glieder der Alaungruppe und das Steinsalz sind besonders häufig untersuchte Kristallarten, mag es sich um das Weiterwachsen künstlich angeschliffener Kugeln oder von ebenflächig begrenzten Kristallen handeln, deren Dimensionen das Keimstadium überschritten haben. In letzteren Fällen läßt

1 K. S pa n g e $\mathrm{n}$ b e r g, Wachstum und Auflösung der Kristalle, in: Hdwörterb. d. Naturw. X, S. 362-401, Jena 1935.

2 K. S p a n g e n b e rg, Kristallwachstum, in: Naturforschung und Medizin in Deutschland 1939-1946, Bd. 49. Mineralogie, S. 221-251, Wiesbaden 1948.

3 P. Nig g li, Lehrbuch d. Mineralogie u. Kristallchemie, I, S. 370 ff., Bornträger 1941. sich oft die Beobachtung machen, daß Kristallflächen mit niedrigen Indices, seien es natürlich gewachsene Flächen cder Spaltflächen, sich beim Wachstum parallel mit sich selbst verschieben. Die Geschwindigkeit, mit der sich eine solche Kristallfläche parallel mit sich selbst verschiebt, nennen wir ihre Wachstums- oder Verschiebungsgeschwindigkeit $v_{(\mathrm{hkl})}$.

Die Beliebtheit der Glieder der Alaungruppe und des Steinsalzes mag darin begründet sein, daß es ohne Schwierigkeit gelingt, mehr oder weniger stark übersättigte, wäßrige Lösungen herzustellen, aus welchen Kristalle leicht gezüchtet werden können. Die auftretenden Kristallflächen sind durch einfache Symmetriegesetze miteinander verknüpft. Endlich zeigen ihre Flächen Wachstumsgeschwindigkeiten, welche so groß sind, daß die Dickenzunahme einfach mit dem Schraubenmikrometer, wie oft in der Literatur angegeben, gemessen werden kann, ohne daß die Ergebnisse durch die diesem Verfahren anhaftende Meßfehlergrenze wesentlich beeinträchtigt werden. 